\title{
ON A MODIFIED HYERS-ULAM STABILITY OF HOMOGENEOUS EQUATION
}

\author{
SOON-MO JUNG \\ Mathematical Part \\ College of Science \& Technology \\ Hong-Ik University \\ 339-800 Chochiwon, SOUTH KOREA
}

(Received February 21, 1996)

ABSTRACT. In this paper, a generalized Hyers-Ulam stability of the homogeneous equation shall be proved, i.e., if a mapping $f$ satisfies the functional inequality $\left\|f(y x)-y^{k} f(x)\right\| \leq \varphi(x, y)$ under suitable conditions, there exists a unique mapping $T$ satisfying $T(y x)=y^{k} T(x)$ and $\|T(x)-f(x)\| \leq \Phi(x)$

KEY WORDS AND PHRASES: Functional equation, homogeneous equation, stability.

1991 AMS SUBJECT CLASSIFICATION CODES: 39B72, 39B52.

\section{INTRODUCTION}

It is well-known that if a real-valued mapping $f$ defined on non-negative real numbers is a solution of the homogeneous equation, i.e. if $f$ satisfies

$$
f(y x)=y^{k} f(x),
$$

where $k$ is a given real number, then $f(x)=c x^{k}$ for some $c \in \mathbb{R}$.

In this note, we shall investigate a generalized Hyers-Ulam stability of the homogeneous equation (1.1) with extended domain and range by using ideas from the paper of Gávruta [1].

Let $(X,+, \cdot)$ be a field and $(X,+,\|\|)$ a real Banach space. In addition, we assume $\|x y\|=\|x\|\|y\|$ for all $x, y \in X$. For convenience, we write $x^{2}, x^{3}, \cdots$ instead of $x \cdot x,(x \cdot x) \cdot x, \cdots$. If there is no confusion we use 0 and 1 to denote the 'zero-element' and the unity (the neutral element with respect to ' ' ') in $X$, respectively. By $x^{-1}$ we denote the multiplicatively inverse element of $x$. Suppose $k$ is a natural number. Let $\varphi: X \times X \rightarrow[0, \infty)$ be a mapping such that

$$
\Phi_{z}(x)=\sum_{j=0}^{\infty}\|z\|^{-(j+1) k} \varphi\left(z^{\jmath} x, z\right)<\infty
$$

or

$$
\tilde{\Phi}_{z}(x)=\sum_{j=0}^{\infty}\|z\|^{j k} \varphi\left(z^{-(\jmath+1)} x, z\right)<\infty
$$

for some $z \in X$ with $\|z\|>1$ and all $x \in X$. Moreover, we assume that 


$$
\left\{\begin{array}{ll}
\Phi_{z}\left(w^{n} x\right)=o\left(\|w\|^{n k}\right) & \text { if } \left.\Phi_{z}(x)<\infty\right) \\
\tilde{\Phi}_{z}\left(w^{n} x\right)=o\left(\|w\|^{n k}\right) & \text { (if } \left.\tilde{\Phi}_{z}(x)<\infty\right)
\end{array},\right.
$$

as $n \rightarrow \infty$, for some $w \in X$ and all $x \in X$. Let a mapping $f: X \rightarrow X$ satisfy

$$
\left\|f(y x)-y^{k} f(x)\right\| \leq \varphi(x, y)
$$

and

$$
\begin{cases}\varphi\left(z^{n} x, y\right)=o\left(\left\|f\left(z^{n} x\right)\right\|\right) \text { as } n \rightarrow \infty & \text { (if } \left.\Phi_{z}(x)<\infty\right) \\ \varphi\left(z^{-n} x, y\right)=o\left(\left\|f\left(z^{-n} x\right)\right\|\right) \text { as } n \rightarrow \infty & \text { (if } \left.\tilde{\Phi}_{z}(x)<\infty\right)\end{cases}
$$

for all $x$ and $y \neq 0$ in $X$. If (1.3) holds true then we further assume $f(0)=0$. Our main result is the following theorem.

THEOREM. There exists a unique mapping $T: X \rightarrow X$ satisfying (1.1) and

$$
\|T(x)-f(x)\| \leq\left\{\begin{array}{ll}
\Phi_{z}(x) & \text { (if } \left.\Phi_{z}(x)<\infty\right) \\
\tilde{\Phi}_{z}(x) & \text { (if } \left.\tilde{\Phi}_{z}(x)<\infty\right)
\end{array},\right.
$$

for all $x \in X$

\section{PROOF OF THEOREM}

We use induction on $n$ to prove

$$
\left\|y^{-n k} f\left(y^{n} x\right)-f(x)\right\| \leq \sum_{j=0}^{n-1}\|y\|^{-(\jmath+1) k} \varphi\left(y^{j} x, y\right)
$$

for any $n \in \mathbb{N}$. By (1.5), it is clear for $n=1$. If we assume that (2.1) is true for $n$, we get for $n+1$

$$
\begin{aligned}
\left\|y^{-(n+1) k} f\left(y^{n+1} x\right)-f(x)\right\| & \leq\|y\|^{-(n+1) k}\left\|f\left(y y^{n} x\right)-y^{k} f\left(y^{n} x\right)\right\|+\left\|y^{-n k} f\left(y^{n} x\right)-f(x)\right\| \\
& \leq\|y\|^{-(n+1) k} \varphi\left(y^{n} x, y\right)+\sum_{j=0}^{n-1}\|y\|^{-(\jmath+1) k} \varphi\left(y^{\jmath} x, y\right) \\
& =\sum_{\jmath=0}^{n}\|y\|^{-(\jmath+1) k} \varphi\left(y^{\jmath} x, y\right)
\end{aligned}
$$

by using (1.5) and (2.1).

(a) First, we assume that $\Phi_{z}(x)<\infty$ for some $z \in X$ with $\|z\|>1$ and all $x \in X$ Let $n>m>0$. It then follows from (2.1) and (1.2) that

$$
\begin{aligned}
\left\|z^{-n k} f\left(z^{n} x\right)-z^{-m k} f\left(z^{m} x\right)\right\| & =\|z\|^{-m k}\left\|z^{-(n-m) k} f\left(z^{n-m} z^{m} x\right)-f\left(z^{m} x\right)\right\| \\
& \leq\|z\|^{-m k} \sum_{j=0}^{n-m-1}\|z\|^{-(\jmath+1) k} \varphi\left(z^{\jmath} z^{m} x, z\right) \\
& =\sum_{j=m}^{n-1}\|z\|^{-(j+1) k} \varphi\left(z^{j} x, z\right) \rightarrow 0 \text { as } m \rightarrow \infty .
\end{aligned}
$$

Therefore, $\left(z^{-n k} f\left(z^{n} x\right)\right)$ is a Cauchy sequence. Since $X$ is a Banach space, we may define

$$
T(x)=\lim _{n \rightarrow \infty} z^{-n k} f\left(z^{n} x\right)
$$

for all $x \in X$. From the definition of $T,(1.2)$ and (2.1) we can easily verify the truth of the first relation in (1.7).

Suppose $x$ and $y \neq 0$ to be arbitrary elements of $X$. By (2.1) we have

$$
\left\|y^{-k} f\left(y z^{n} x\right)-f\left(z^{n} x\right)\right\| \leq\|y\|^{-k} \varphi\left(z^{n} x, y\right) .
$$


It follows from the inequality just above and (1.6) that

$$
\left\|f\left(z^{n} x\right)^{-1} y^{-k} f\left(y z^{n} x\right)-1\right\| \leq\|y\|^{-k}\left\|f\left(z^{n} x\right)\right\|^{-1} \varphi\left(z^{n} x, y\right) \rightarrow 0 \text { as } n \rightarrow \infty .
$$

Hence, it holds

$$
\lim _{n \rightarrow \infty} f\left(z^{n} x\right)^{-1} y^{-k} f\left(y z^{n} x\right)=1 .
$$

By (2.2) we can show that for all $x$ and $y \neq 0$ in $X$

$$
\begin{aligned}
T(y x) & =\lim _{n \rightarrow \infty} z^{-n k} f\left(z^{n} y x\right) \\
& =y^{k} \lim _{n \rightarrow \infty} z^{-n k} f\left(z^{n} x\right) \lim _{n \rightarrow \infty} f\left(z^{n} x\right)^{-1} y^{-k} f\left(z^{n} y x\right) \\
& =y^{k} T(x) .
\end{aligned}
$$

Besides, it is not difficult to show that $T(0)=0$. Hence, $T(y x)=y^{k} T(x)$ holds true for all $x, y \in X$

Let $U: X \rightarrow X$ be another mapping which fulfills (1.1) and (1.7). By using (1.1), (1 7) and (1.4) we get

$$
\|T(x)-U(x)\|=\|w\|^{-n k}\left\|T\left(w^{n} x\right)-U\left(w^{n} x\right)\right\| \leq 2\|w\|^{-n k} \Phi_{z}\left(w^{n} x\right) \rightarrow 0 \text { as } n \rightarrow \infty .
$$

Hence, it is clear that $T(x)=U(x)$ for all $x \in X$.

(b) Now, we consider the case $\tilde{\Phi}_{z}(x)<\infty$ for some $z \in X$ with $\|z\|>1$ and all $x \in X$ By replacing $x$ in (2.1) with $y^{-n} x$ we get

$$
\left\|f(x)-y^{n k} f\left(y^{-n} x\right)\right\| \leq \sum_{\jmath=0}^{n-1}\|y\|^{\jmath k} \varphi\left(y^{-(\jmath+1)} x, y\right)
$$

for any $n \in \mathbb{N}$. As in part (a), if $n>m>0$ then we obtain

$$
\left\|z^{n k} f\left(z^{-n} x\right)-z^{m k} f\left(z^{-m} x\right)\right\| \leq \sum_{\jmath=m}^{n-1}\|z\|^{\jmath k} \varphi\left(z^{-(\jmath+1)} x, z\right) \rightarrow 0 \text { as } m \rightarrow \infty,
$$

by using (2.3) and (1.3). We may define

$$
T(x)=\lim _{n \rightarrow \infty} z^{n k} f\left(z^{-n} x\right)
$$

for all $x \in X$. Hence, the second inequality in (1.7) is obvious in view of (2.3)

For arbitrary $x$ and $y \neq 0$ in $X$, it follows from (2.1) and (1.6) that

$$
\lim _{n \rightarrow \infty} f\left(z^{-n} x\right)^{-1} y^{-k} f\left(y z^{-n} x\right)=1
$$

as in part (a) above. By using (2.4), we get for $x$ and $y \neq 0$ in $X$

$$
\begin{aligned}
T(y x) & =\lim _{n \rightarrow \infty} z^{n k} f\left(z^{-n} y x\right) \\
& =y^{k} \lim _{n \rightarrow \infty} z^{n k} f\left(z^{-n} x\right) \lim _{n \rightarrow \infty} f\left(z^{-n} x\right)^{-1} y^{-k} f\left(y z^{-n} x\right) \\
& =y^{k} T(x) .
\end{aligned}
$$

Since $f(0)=0$ is assumed in the case of $\tilde{\Phi}_{z}(x)<\infty$, it also holds $T(y x)=y^{k} T(x)$ for $y=0$

The uniqueness of $T$ can be proved as in (a).

\section{EXAMPLES}

EXAMPLE 1. Let $\varphi(x, y)=\delta+\theta\|x\|^{a}\|y\|^{b}(\delta \geq 0, \theta \geq 0,0 \leq a<k, b \geq 0)$ be given in the functional inequality (1.5). If a mapping $f: X \rightarrow X$ satisfies the first condition in (1.6) then there exists a unique mapping $T: X \rightarrow X$ fulfilling (1.1) and 


$$
\|\mathrm{T}(x)-f(x)\| \leq \delta\left(\|z\|^{k}-1\right)^{-1}+\theta\|z\|^{b}\left(\|z\|^{k}-\|z\|^{a}\right)^{-1}\|x\|^{a}
$$

for any $x, z \in X$ with $\|z\|>1$. In particular, if $\delta>0$ and $\theta=0$ then $f$ itself satisfies (1.1).

EXAMPLE 2. Assume that $\varphi(x, y)=\theta\|x\|^{a}\|y\|^{b}(\theta \geq 0, a>k, b \geq 0)$ is given in the functional inequality (1.5) If a mapping $f: X \rightarrow X$ satisfies the second condition in (1.6) then there exists a unique mapping $T: X \rightarrow X$ which satisfies (1.1) and

$$
\|T(x)-f(x)\| \leq \theta\|z\|^{b}\left(\|z\|^{a}-\|z\|^{k}\right)^{-1}\|x\|^{a}
$$

for all $x, z \in X$ with $\|z\|>1$.

If $\varphi(x, y)=\theta\|x\|^{k} g(\|y\|)$ for some mapping $g:[0, \infty) \rightarrow[0, \infty)$ then our method to get stability for the homogeneous equations (1 1 ) cannot be applied. By modifying an example in the paper of Rassias and Šemrl [2] we shall introduce a mapping $f: \mathbb{R} \rightarrow \mathbb{R}$ satisfying (1.5) and (1.6) with some $\varphi$ and such that $|f(x)||x|^{-k}$ (for $x \neq 0$ ) is unbounded.

EXAMPLE 3. Let us define $f(x)=x^{k} \log |x|$ for $x \neq 0$ and $f(0)=0$. Then $f$ satisfies (1.5) and both conditions of (1..6) with $\varphi(x, y)=|x|^{k}|y|^{k}|\log | y||(y \neq 0)$ and $\varphi(x, 0)=0$, even though $\varphi$ satisfies neither (1.2) nor (1.3). In this case we can expect no analogy to the results of Example 1 and 2 Really, it holds

$$
\lim _{n \rightarrow \infty}|T(x)-f(x)||x|^{-k}=\infty
$$

for each mapping $T: \mathbb{R} \rightarrow \mathbb{R}$ fulfilling (1.1).

\section{REFERENCES}

[1] GẢVRUTA, P., A generalization of the Hyers-Ulam-Rassias stability of approximately additive mappings, J. Math. Anal. Appl. 184 (1994), 431-436.

[2] RASSIAS, TH.M. and ŚEMRL, P., On the behavior of mappings which do not satisfy Hyers-Ulam stability, Proc. Amer. Math. Soc. 114 (1992), 989-993. 




Advances in

Operations Research

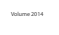



\section{The Scientific} World Journal
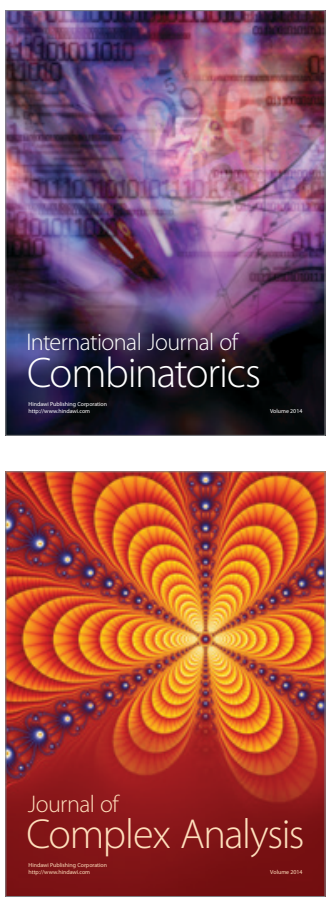

International Journal of

Mathematics and

Mathematical

Sciences


Journal of

Applied Mathematics
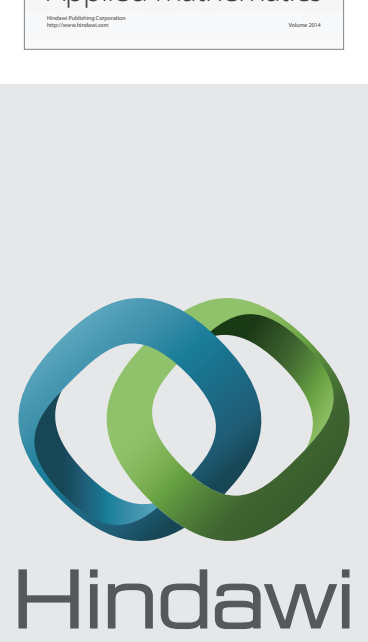

Submit your manuscripts at http://www.hindawi.com


Mathematical Problems in Engineering
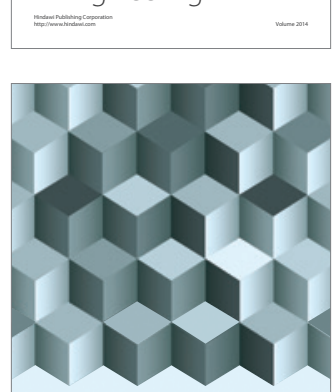

Journal of

Function Spaces
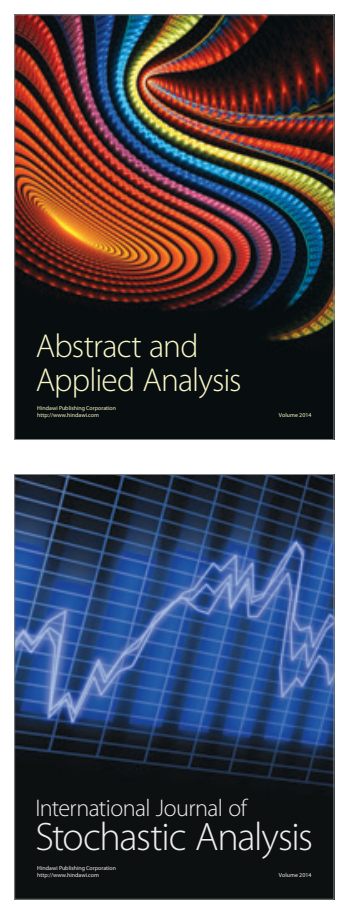

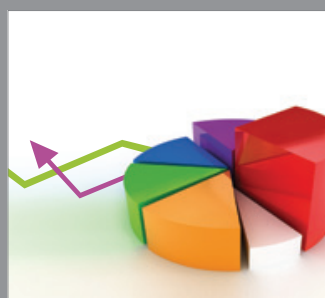

ournal of

Probability and Statistics

Promensencen
